\title{
Symbol Reliability Estimation Using the Bidirectional Viterbi Decoding Algorithm
}

\author{
Masato Tajima Keiji Takida Zenshiro Kawasaki \\ Dept. of Intellectual Information Systems Engineering, \\ Faculty of Engineering, 3190 Gofuku, Toyama 930, Japan \\ E-mail : tajima@ecs.toyama-u.ac.jp
}

\begin{abstract}
In this paper, we first review the Soft-BIVA based on the bidirectional Viterbi algorithm (BIVA). Next, we apply the Soft-BIVA to QLI codes under the assumption that a Scarce State Transition Viterbi decoder is used. Then the symbol reliability values are derived by applying the Soft-BIVA to the main decoder. Moreover, it is shown that in the case of QLI codes, the symbol reliability is also obtained using bidirectional syndrome decoding in the syndrome trellis.
\end{abstract}

\section{Introduction}

We restrict the following discussion to a binary rate $1 / \mathrm{n}$ convolutional code specified by a polynomial generator matrix $G(D)$ to preserve clarity. Let $u=\left\{u_{k}\right\}$ be a transmitted information sequence. Also denote by $z=\left\{Z_{k}\right\}$ the received sequence corresponding to a convolutionally coded sequence $\mathrm{w}(\mathrm{D})=\mathrm{u}(\mathrm{D}) \mathrm{G}(\mathrm{D})$. Then symbol-by-symbol MAP decoding is accomplished by maximizing the joint probability $\mu\left(\mathrm{u}_{\mathrm{k}}, \mathrm{z}\right)$ for each time $\mathrm{k}$. The scheme is named the BCJR algorithm after the inventors [1]. This BCJR algorithm requires a "backward" as well as a forward recursion and consequently storage of all data. Moreover, it has to deal with not path metrics but the exact path probabilities. Hence its computational complexity is high. However, it can generate reliability informaion on the decoded symbols. At the beginning the BCJR algorithm did not attract much attention for the reason of not having a particular advantage with respect to the bit error rate compared with the Viterbi algorithm (VA) [2]. But in 1993 the "turbo-codes" were proposed [3] and the situation has changed. Actually, reliability information on the decoded symbols plays an essential role in turbo decoding. Since then the BCIR algorithm has been attracting a great deal of attention [4], [5]. In particular, reduction of the computational complexity of the original BCJR algorithm is one of the main problems to be solved.

On the other hand, apart from the above circumstances, it has been known that a convolutionally coded sequence corresponding to a frame of information bits terminated by tail bits can be decoded from both directions simultaneously (i.e., bidirectional Viterbi decoding [6], [7]). Since in this method the states on the maximum-likelihood (ML) path are identified by joining the forward and backward decoders, the authors thought that bidirectional Viterbi decoding must have close relation to the BCJR algorithm which also needs both forward and backward recursions. As a result, we have shown in [8] that an approximate value of the $\log$-likelihood ratio $\Lambda_{\mathrm{k}} \equiv \ln \mid \mu$ $\left(u_{k}=0 \mid z\right) / \mu\left(u_{k}=1 \mid z\right) \mid$ (i.e., reliability information on $\left.u_{k}\right)$ can be obtained using bidirectional Viterbi decoding.

Now, we divide the time-k states $\left\{\mathrm{s}_{\mathrm{k}}\right\}$ in the trellis into two groups:

$$
\begin{aligned}
& \left\{\mathrm{s}_{\mathrm{k}}{ }^{+}=\left(\mathrm{u}_{\mathrm{k}-\nu+1}, \cdots, \mathrm{u}_{\mathrm{k}-1}, \mathrm{u}_{\mathrm{k}}=0\right)\right\}, \\
& \left\{\mathrm{s}_{\mathrm{k}}{ }^{-}=\left(\mathrm{u}_{\mathrm{k}-\nu+1}, \cdots, \mathrm{u}_{\mathrm{k}-1}, \mathrm{u}_{\mathrm{k}}=1\right)\right\},
\end{aligned}
$$

where $\nu$ is the memory length of G(D). If the forward and backward decoders join at time $\mathrm{k}$, the best path $\mathrm{x}^{\mathrm{*}}\left(\mathrm{S}_{\mathrm{k}}\right)$ which passes through each state $s_{\mathrm{k}}$ in the trellis is determined. We denote by $\mathrm{m}^{*}\left(\mathrm{~s}_{\mathrm{k}}\right)$ the path metric (i.e., $\log$-likelihood) of $x^{*}\left(s_{k}\right)$. Then it is shown that

$$
\begin{aligned}
& \rho_{\mathrm{k}}{ }^{*} \equiv \max _{\mathrm{u}_{\mathrm{k}-\nu+1}, \cdots, \mathrm{u}_{\mathrm{k}-1}}\left\{\mathrm{~m}^{\left.*{ }^{*}\left(\mathrm{~s}_{\mathrm{k}}{ }^{+}\right)\right\}}\right. \\
& \quad-\max _{\mathrm{u}_{\mathrm{k}-\nu+1}, \cdots, \mathrm{u}_{\mathrm{k}-1}}\left\{\mathrm{~m}^{*}\left(\mathrm{~s}_{\mathrm{k}}{ }^{-}\right)\right\}
\end{aligned}
$$

is a good approximation to $\Lambda_{\mathrm{k}}$. In the following, we call the above scheme the bidirectional Viterbi decoding algorithm with soft-outputs (Soft-BIVA). (A similar idea is given in $[9]$.) 
In this paper, we first state the basic properties of the Soft-BIVA. In particular, we examine the relation between the Soft-BIVA and the Sott-Output Viterbi Algorithm (SOVA) proposed by Hagenauer et al. [10]. Next, we apply the Soft-BIVA to Quick Look-In (QLI) codes under the assumption that a Scarce State Transition (SST) Viterbi decoder [11] is used. Then the symbol reliability values are derived by applying the Soft-BIVA to the main decoder. Moreover, it is shown that in the case of QLI codes, the symbol reliability is also obtained using bidirectional syndrome decoding in the syndrome trellis. Here note that the syndrome sequence must be generated in time-reversed order for backward syndrome decoding. It is shown that the time-reversed syndrome sequence can be generated using a generator matrix of the "reciprocal dual code" [12] of the original code.

In the following, a BPSK modulation/demodulation is assumed and a coded sequence is transmitted symbol by symbol over a memoryless AWGN channel. The $j$-th received data can be modeled as $z_{j}=y_{j} \sqrt{ }\left(2 E_{s} / N_{0}\right)+n_{j}$, where $y_{j}= \pm 1$ depending on whether the code symbol is 0 or $1, n_{j}$ denotes a zero-mean unit variance Gaussian random variable, $E_{s}$ is the signal energy per coded bit, and $\mathrm{N}_{0}$ is one-sided power spectral density of the noise. Terminated data transmission is also assumed.

\section{Review of the Soft-BIVA}

\subsection{Soft-BIVA}

For evaluating the symbol reliability, all the paths which pass through $\mathrm{s}_{\mathbf{k}}{ }^{ \pm}$are taken into consideration in the BCIR algorithm. On the other hand, the Soft-BIVA estimates the symbol reliability using only the best path which passes through $s_{k}{ }^{ \pm}$. (Note that the Soft-BIVA is based on the Viterbi algorithm.) Now let

$$
\begin{aligned}
& \beta_{0} \equiv \Sigma_{u_{k-\nu+1}, \cdots, u_{k-1}} \mu\left(z \mid x^{*}\left(s_{k}{ }^{+}\right)\right), \\
& \beta_{1} \equiv \Sigma_{u_{k-\nu+1}, \cdots, u_{k-1}} \mu\left(z \mid x^{*}\left(s_{k}{ }^{-}\right)\right) .
\end{aligned}
$$

Then it is shown that $\ln \left[\beta_{0} / \beta_{1}\right]$ is approximated by $\rho_{\mathrm{k}}$ " [8].

Here let $\mathrm{s}_{\mathrm{k}}{ }^{\wedge}=\left(\mathrm{u}_{\mathrm{k}-\nu+1} \hat{}, \cdots, \mathrm{u}_{\mathrm{k}-1} \hat{}{ }^{, \mathrm{u}_{\mathrm{k}}} \hat{}{ }^{\prime}\right)$ be the state at time $\mathrm{k}$ which the $\mathrm{ML}$ path passes through, where the ML path is determined when the forward and backward decoders join. Since $m^{*}\left(s_{k}{ }^{\wedge}\right)=\max _{S_{k}} m^{*}\left(S_{k}\right), \quad \rho_{k}{ }^{*}$ is expressed as

$$
\rho_{\mathrm{k}}{ }^{*}=\Delta_{\mathrm{k}}{ }^{*} \cdot\left(1-2 \mathrm{u}_{\mathrm{k}}{ }^{\wedge}\right)
$$

where

$$
\begin{aligned}
& \Delta_{\mathrm{k}}{ }^{*} \equiv \mathrm{m}^{*}\left(\mathrm{~s}_{\mathrm{k}}{ }^{\wedge}\right) \\
& \quad-\max _{\mathrm{u}_{\mathrm{k}-\nu+1}, \cdots, \mathrm{u}_{\mathrm{k}-1}}\left\{\mathrm{~m}^{*}\left(\mathrm{u}_{\mathrm{k}-\nu+1}, \cdots, \mathrm{u}_{\mathrm{k}-1}, 1-\mathrm{u}_{\mathrm{k}}{ }^{\wedge}\right)\right\} \\
& (\geqq 0) .
\end{aligned}
$$

Remark : If $\left\{\mathrm{s}_{\mathrm{k}}{ }^{+}\right\}=\phi$ or $\left\{\mathrm{s}_{\mathrm{k}}{ }^{-}\right\}=\phi$, we set $\Delta_{\mathrm{k}}{ }^{*}=\infty$, where $\phi$ is the empty set.

Under these conditions, the Soft-BIVA is defined as follows $[8]$ :

\section{$<$ Soft-BIVA $>$}

$\left\lceil\right.$ Evaluate the metric of the best path $x^{*}\left(s_{k}\right)$ which passes through each state $s_{k}$ of the trellis at time $k$, where $x^{*}\left(s_{k}\right)$ is determined when the forward and backward decoders join at time $\mathrm{k}$. Then identify the ML-state $\mathrm{s}_{\mathrm{k}}{ }^{\wedge}=\left(\mathrm{u}_{\mathrm{k}-\nu+1}{ }^{\wedge}, \cdots\right.$, $\mathrm{u}_{\mathrm{k}}{ }^{\wedge}$ ) which the ML path passes through at time $\mathrm{k}$. At the same time, calculate the reliability of the symbol $u_{k}$ " using $\Delta_{\mathrm{k}}{ }^{*}$.」

In the Soft-BIVA, only the best path which passes through each state of the trellis is considered. Here, we examine the variation of $\rho_{\mathrm{k}}{ }^{*}$ in the case where the second best paths are also taken into consideration in addition to the best paths. Let

$$
\begin{aligned}
& \beta_{0}^{\prime} \equiv \Sigma_{\mathrm{u}_{\mathrm{k}-\nu+1}, \cdots, \mathrm{u}_{\mathrm{k}-1}}\left\{\mu\left(\mathrm{z} \mid \mathrm{x}^{* *}\left(\mathrm{~s}_{\mathrm{k}}{ }^{+}\right)\right)+\mu\left(\mathrm{z} \mid \mathrm{x}^{* * *}\left(\mathrm{~s}_{\mathrm{k}}{ }^{+}\right)\right)\right\}, \\
& \beta_{1}^{\prime} \equiv \Sigma_{\mathrm{u}_{\mathrm{k}-\nu+1}, \cdots, \mathrm{u}_{\mathrm{k}-1}}\left\{\mu\left(\mathrm{z} \mid \mathrm{x}^{*}\left(\mathrm{~s}_{\mathrm{k}}{ }^{-}\right)\right)+\mu\left(\mathrm{z} \mid \mathrm{x}^{* * *}\left(\mathrm{~s}_{\mathrm{k}}{ }^{-}\right)\right)\right\},
\end{aligned}
$$

where $x^{* * *}\left(s_{k}\right)$ denotes the second best path which passes through $\mathrm{s}_{\mathbf{k}}$. Next, we evaluate the difference $\rho^{(1)} \equiv \ln \mid \beta_{0}^{\prime}$ $/ \beta_{1}^{\prime}|-\ln | \beta_{0} / \beta_{1} \mid$. Let $\Delta^{ \pm}$be

$$
\begin{aligned}
& \Delta^{ \pm} \equiv \max _{\mathrm{u}_{\mathrm{k}-\nu+1}, \cdots, \mathrm{u}_{\mathrm{k}-1}}\left\{\mathrm{~m}^{\prime \prime}\left(\mathrm{s}_{\mathrm{k}}{ }^{ \pm}\right)\right\} \\
& \quad-\max _{\mathrm{u}_{\mathrm{k}-\nu+1}, \cdots, \mathrm{u}_{\mathrm{k}-1}}\left\{\mathrm{~m}^{* * *}\left(\mathrm{~s}_{\mathrm{k}}{ }^{ \pm}\right)\right\}(\geqq \mathrm{A}>0),
\end{aligned}
$$

where $m^{m * *}\left(s_{k}\right)$ denotes the metric of the second best path $\mathrm{x}^{\mathrm{m}}\left(\mathrm{s}_{\mathrm{k}}\right)$. Then it is shown that if $\mathrm{e}^{-\mathrm{A}}$ is sufficiently small, $\rho^{(1)}$ is approximated by

$$
\left.\left.\rho^{(1)} \fallingdotseq \exp \mid-\Delta^{+}\right]-\exp \mid-\Delta^{-}\right]
$$

Hence, if the above assumption holds, we can conclude that the difference $\rho^{(1)}$ is small. 


\subsection{Relation between Soft-BIVA and SOVA}

In this section, we discuss the relation between the Soft-BIVA and the SOVA. In the SOVA, the reliability of the symbol $u_{k}$ ' on the ML path is evaluated by the process in which the $\mathrm{ML}$ path has survived through competitions with the discarded paths [10]. Actually, the reliability of the symbol $u_{k}{ }^{\wedge}$ is defined as

$$
\Delta_{\mathrm{k}}{ }^{\mathrm{H}} \equiv \min _{1} \leqq \mathrm{~d} \leqq \delta_{1} \Delta_{\mathrm{k}}{ }^{\mathrm{d}},
$$

where $\Delta_{k}{ }^{d}$ denotes the metric difference between the ML path and the discarded path at time $\mathrm{k}+\mathrm{d}\left(\leqq \mathrm{k}+\delta_{1}, \delta_{1}\right.$ is the truncation length of a survivor).

Remark : If the discarded path at time $\mathrm{k}^{+} \mathrm{d}$ passes through a state $\left(\cdots, u_{k}{ }^{\wedge}\right)$ at time $k$, we set $\Delta_{k}{ }^{d}=\infty$.

Here, we assume that $\delta_{1}$ is large enough so that all survivors have been merged with sufficiently high probability (we rewrite $\delta_{1}$ as $\delta$ ). Then it is shown that the inequality:

$$
\Delta_{\mathrm{k}}{ }^{{ }} \leqq \Delta_{\mathrm{k}}{ }^{\mathrm{H}}
$$

holds [8]. Furthermore, let $\mathrm{s}_{\mathrm{k}}{ }^{*} \equiv\left(\mathrm{u}_{\mathrm{k}-{ }_{\nu}+1}{ }^{*}, \cdots, \mathrm{u}_{\mathrm{k}-1}{ }^{*}, 1-\mathrm{u}_{\mathrm{k}}{ }{ }\right)$ be the state at which $\Delta_{k}{ }^{*}$ is attained and take notice of the best path $\mathrm{x}^{\#}$ which passes through the state $\mathrm{s}_{\mathrm{k}}{ }^{*}$. We also assume that $\mathrm{x}^{\#}$ has merged with the ML path at $\mathrm{k}+\mathrm{d}^{\prime}$ ( $\leqq$ $k+\delta)$. In this case, if $x^{\#}$ has contended with the ML path at time $\mathrm{k}^{+} \mathrm{d}^{\prime}$, then it is shown that $\Delta_{\mathrm{k}}{ }^{*}=\Delta_{\mathrm{k}}{ }^{\mathrm{H}}$ holds [8].

In the SOVA, the probability of decision error $\mathrm{P}_{\mathrm{e}} \equiv \mu$ $\left(\mathrm{u}_{\mathrm{k}}{ }^{\wedge} \neq \mathrm{u}_{\mathrm{k}}\right)$ is approximated by $\mathrm{P}_{\mathrm{e}}{ }^{\mathrm{H}}=1 /\left(1+\exp \mid \Delta_{\mathrm{k}}{ }_{\mathrm{H}} \mathrm{l}\right)$. Similarly, it is shown that $\mathrm{P}_{\mathrm{e}}$ is approximated by $\mathrm{P}_{e}^{*}=1 /\left(1+\exp \left[\Delta_{\mathrm{k}}{ }^{*}\right]\right)$ in the Soft-BIVA.Then it follows that $\mathrm{P}_{\mathrm{e}}^{\mathrm{H}} \leqq \mathrm{P}_{\mathrm{e}}{ }^{*}$ from the relation $\Delta_{\mathrm{k}}{ }^{*} \leqq \Delta_{\mathrm{k}}{ }^{\mathrm{H}}$.

\subsection{Simulation Results}

Simulations were conducted to evaluate the relation between the Soft-BIVA and the SOVA. A rate- $1 / 2$ code with generator matrix $G_{1}=\left(1+D^{2}, 1+D+D^{2}\right)$ was used. For applying the SOVA(or Soft-BIVA), the conventional integer code symbol metrics $\mathrm{m}_{\mathrm{j}}{ }^{\prime}(0 \sim 7)$ were used according to the formula $m_{j}{ }^{\prime}=2 \times z_{j}{ }^{\prime \prime} \times y_{j}+3.5$, where $z_{j}{ }^{*}$ is the median of the interval to which the received data $z_{j}$ belongs. We set $\delta{ }_{1}=36$. The length of an information squence is about $10^{7}$. Then it has been found that the percentage of $\Delta_{\mathrm{k}}{ }^{*}=\Delta_{\mathrm{k}}{ }^{\mathrm{H}}$ is $60 \sim 85 \%$ for $\mathrm{E}_{\mathrm{b}} / \mathrm{N}_{0}$ between 0 and $6 \mathrm{~dB}[8]$. Table 1 shows the average $\Delta_{\mathrm{k}}{ }^{\mathrm{H}}$ and $\Delta_{\mathrm{k}}{ }$ for correct and incorrect VA outputs (- denotes the average). It is shown that the Soft-BIVA generates slightly smaller symbol reliability values compared with the SOVA. Simulations were also conducted to compare $\mathrm{P}_{e}^{\mathrm{H}}$ and $\mathrm{P}_{e}{ }^{*}$ with $\mathrm{P}_{\mathrm{e}}$, where $\mathrm{P}_{\mathrm{e}}$ was calculated using the $B C J R$ algorithm. Table 2 shows their averages for incorrect VA outputs (cf., $[5])$. It is confirmed that the estimate $\mathrm{P}_{\mathrm{e}}$ * derived from the Soft-BIVA is nearer to $P_{e}$ compared with $\mathrm{P}_{e}^{\mathrm{H}}$.

Table 1 Average $\Delta_{k}{ }^{H}$ and $\Delta_{k}{ }^{*}$ for correct and incorrect VA outputs

\begin{tabular}{|c|c|c|r|c|}
\hline \multirow{2}{*}{$\begin{array}{c}\mathrm{E}_{\mathrm{b}} / \mathrm{N}_{0} \\
(\mathrm{~dB})\end{array}$} & \multicolumn{2}{|c|}{$\overline{\Delta_{\mathrm{k}}{ }^{\mathrm{H}}}$ (SOVA) } & $\overline{\Delta_{\mathrm{k}}{ }^{*}}$ (Soft-BIVA) \\
\cline { 2 - 5 } & correct & incorrect & correct & incorrect \\
\hline 0.0 & 11.64 & 3.75 & 9.75 & 2.92 \\
0.5 & 12.81 & 3.61 & 10.96 & 2.84 \\
1.0 & 14.11 & 3.44 & 12.34 & 2.74 \\
1.5 & 15.53 & 3.27 & 13.85 & 2.64 \\
2.0 & 17.04 & 3.11 & 15.48 & 2.55 \\
2.5 & 18.61 & 2.90 & 17.17 & 2.41 \\
3.0 & 20.20 & 2.73 & 18.90 & 2.30 \\
3.5 & 21.78 & 2.51 & 20.63 & 2.18 \\
4.0 & 23.32 & 2.32 & 22.31 & 2.05 \\
\hline
\end{tabular}

Table 2 Average $\mathrm{P}_{e}{ }_{\mathrm{H}}, \mathrm{P}_{\mathrm{e}}^{*}$ and $\mathrm{P}_{\mathrm{e}}$ for incorrect VA outputs

\begin{tabular}{|c|c|c|c|}
\hline $\mathrm{E}_{\mathrm{b}} / \mathrm{N}_{0}(\mathrm{~dB})$ & $\overline{\mathrm{P}_{\mathrm{e}}^{\mathrm{H}}}$ & $\overline{\mathrm{P}_{\mathrm{e}}^{*}}$ & $\overline{\mathrm{P}_{\mathrm{e}}}$ \\
\hline 0.0 & 0.2276 & 0.2477 & 0.2769 \\
0.5 & 0.2272 & 0.2448 & 0.2666 \\
1.0 & 0.2283 & 0.2433 & 0.2595 \\
1.5 & 0.2286 & 0.2412 & 0.2540 \\
2.0 & 0.2293 & 0.2399 & 0.2442 \\
2.5 & 0.2323 & 0.2411 & 0.2367 \\
3.0 & 0.2341 & 0.2413 & 0.2392 \\
3.5 & 0.2354 & 0.2406 & 0.2391 \\
\hline
\end{tabular}

Remark : For evaluating $\mathrm{P}_{\mathrm{e}}^{\mathrm{H}}\left(\mathrm{P}_{\mathrm{e}}^{*}\right)$, the exact loglikelihoods $m_{j}$ are necessary. Since $m_{j}$ is expressed as $m_{j}=$ $\sqrt{ }\left(2 \mathrm{E}_{\mathrm{s}} / \mathrm{N}_{\mathrm{o}}\right) \times \mathrm{z}_{\mathrm{j}} \times \mathrm{y}_{\mathrm{j}}+\eta_{\mathrm{j}}$, integer metrics were transformed to the log-likelihoods by the formula $\Delta_{k}{ }^{H}\left(\Delta_{k}{ }^{*}\right) \times \sqrt{ }$ $\left(2 \mathrm{E}_{\mathrm{s}} / \mathrm{N}_{\mathrm{o}}\right) / 2$. Hence, some errors due to metric quantization are contained in $\mathrm{P}_{\mathrm{e}}^{\mathrm{H}}$ and $\mathrm{P}_{\mathrm{e}}^{*}$. However, they are expected to be small. 


\section{Application of Soft-BIVA to QLI Codes} 3.1 SST Viterbi Decoding of QLI Codes [11], [13]

In this section, we apply the Soft-BIVA to a general rate $1 / 2$, memory length $\nu$, QLI code specified by a polynomial generator matrix:

$$
G=\left(g^{(1)}(D), g^{(2)}(D)\right)\left(g^{(1)}(D)+g^{(2)}(D)=D^{L}, 1 \leqq L \leqq \nu-1\right)
$$

Of course, the results in the preceding section also apply to QLI codes. For QLI codes, however, in addition to conventional Viterbi decoding, SST Viterbi decoding which consists of two decoding steps (i.e., pre-decoding + main decoding) can be applied [11], [13].

Let "Hard" mean hard-decision. Then $\operatorname{Hard}(\mathrm{z}(\mathrm{D}))$ is expressed as $\operatorname{Hard}(z(D))=i(D) G+e(D)$, where $i(D)$ and $e(D)$ are the original information sequence and the error sequence, respectively. Now consider SST Viterbi decoding of G. In this scheme, first, the information sequence $i(D)$ is roughly estimated by

$$
\operatorname{Hard}(z(D)) G^{*-1}=i(D) D^{L}+e(D) G^{*-1},
$$

where $\mathrm{G}^{*-1}=(1,1)^{\mathrm{T}}$ ( $\mathrm{T}$ means transpose) corresponds to pre-decoding. Next, the pre-decoded sequence is re-encoded and then added to the $L$-delayed received sequence $z(D) D^{L}$. The result $z^{\#}$ (D) becomes the input to the main decoder. In particular, the hard-decision input to the main decoder is expressed as

$$
\begin{aligned}
& \operatorname{Hard}\left(z^{\#}(D)\right)=\left(\operatorname{Hard}(z(D)) G^{*-1}\right) G+\operatorname{Hard}(z(D)) D^{\mathrm{L}} \\
& =\left(e(D) G^{*-1}\right) G+e(D) D^{L} \cdots(*) \\
& =e(D)\left(H^{T}, H^{T}\right)=(\zeta(D), \zeta(D))
\end{aligned}
$$

where $H^{T}=\left(g^{(2)}(D), g^{(1)}(D)\right)^{T}$ is the syndrome former corresponding to $G$ and $\zeta$ (D) represents the syndrome sequence. In the main decoder, $u(D) \equiv e(D) G^{*-1}$ (i.e., $u_{k}=e_{k}^{(1)}+e_{k}^{(2)}$ ) is decoded using the Viterbi algorithm (cf., $(*))$. Finally, it is added to the output from the pre-decoder, i.e., $\operatorname{Hard}(z(D)) G^{*-1}=i(D) D^{L}+e(D) G^{*-1}$, to get the original information sequence $i(D) D^{L}$.

\subsection{Application of Soft-BIVA to the Main Decoder}

Let $\left\{\mathrm{i}_{\mathrm{k}}\right\}$ be the original information sequence. Denote by $\left\{u_{k}=e_{k}{ }^{(1)}+e_{k}{ }^{(2}\right\}$ the apparent information sequence for the main decoder. Then the final time-k output from the decoder is expressed as $i_{k-L}=\left(i_{k-L}+u_{k}\right)+u_{k}$. Since $\left(i_{k-L}+u_{k}\right)$ is the output from the pre-decoder (i.e., uniquely determined from the received data), we have

$$
\begin{aligned}
|\ln | \mu\left(\mathrm{i}_{\mathrm{k}-\mathrm{L}}\right. & \left.=0 \mid z) / \mu\left(\mathrm{i}_{\mathrm{k}-\mathrm{L}}=1 \mid \mathrm{z}\right)\right] \mid \\
& =|\ln | \mu\left(\mathrm{u}_{\mathrm{k}}=0 \mid \mathrm{z}\right) / \mu\left(\mathrm{u}_{\mathrm{k}}=1 \mid \mathrm{z}\right)|| .
\end{aligned}
$$

That is, the reliability of the original symbol $i_{k-L}$ is identical to that of $u_{k}$. By the way, since $u_{k}=e_{k}^{(1)}+e_{k}^{(2)}$ is the apparent information symbol for the main decoder, its reliability can be obtained by applying the Soft-BIVA to the main decoder. Note that the application of the Soft-BIVAis justified by the following facts:

1) In the Soft-BIVA, the path metric of the best path which passes through $\mathrm{s}_{\mathrm{k}}{ }^{ \pm}$is used for symbol reliability estimation.

2) For SST Viterbi decoding of QLI codes, metrics can be computed using the conventional method by regarding $\left\{u_{k}\right\}$ as the original information sequence [14].

If these assumptions did not hold, we could not apply the Soft-BIVA to the main decoder in an SST Viterbi decoder.

\section{Symbol Reliability Estimation Using Bidirectional Syndrome Decoding}

It is known that the structure of the main decoder in an SST Viterbi decoder for QLI codes is closely related to syndrome decoding [13]. Then it is quite natural to expect that the reliability of the symbol $i_{k-L}$ can be obtained using bidirectional syndrome decoding.

Again we take a QLI code specified by $G=\left(g^{(1)}(D)\right.$, $\left.g^{(2)}(D)\right)\left(g^{(1)}(D)+g^{(2)}(D)=D^{L}\right)$. The corresponding syndrome former is given by $H^{\mathrm{T}}=\left(g^{(2)}(D), g^{(1)}(D)\right)^{\mathrm{T}}$. We assume that the degree of $g^{(n)}(D)(n=1,2)$ is $\nu$. Also let $\sigma(k)=\left(\sigma_{1}(k)\right.$, $\left.\sigma_{2}(\mathrm{k}), \cdots, \sigma_{\nu}(\mathrm{k})\right)$ be the time- $\mathrm{k}$ state representation of an observer canonical form [15] of the syndrome former $\mathrm{H}^{\mathrm{T}}$. Then the least significant bit $\sigma_{\nu}(\mathrm{k})$ is expressed as $\sigma_{\nu}$ $(\mathrm{k})=\mathrm{e}_{\mathrm{k}}^{(1)}+\mathrm{e}_{\mathrm{k}}^{(2)}$.

Since $\sigma_{\nu}(\mathrm{k})$ is the least significantbit of the state $\sigma(\mathrm{k})$ in the syndrome trellis of $\mathrm{H}^{\mathrm{T}}$, its reliability can be estimated using bidirectional syndrome decoding like the Soft-BIVA. That is, the reliability of $\mathrm{e}_{\mathrm{k}}^{(1)}+\mathrm{e}_{\mathrm{k}}^{(2)}$ is obtained by applying bidirectional syndrome decoding.

Next, we investigate what is corresponding to the reliability of $e_{k}^{(1)}+e_{k}^{(2)}$ in the original information 
sequence. It is known that there exists a one-to-one correspondence between the states of an encoder and those of the corresponding syndrome former [15]. Let an encoder physical state be $\left(i_{k-\nu}, \cdots, i_{k-L}, \cdots, i_{k-1}, i_{k}\right)$. This state is represented by a polynomial $\psi$ in $\mathrm{D}^{-1}$ :

$$
\psi=\mathrm{i}_{\mathrm{k}}+\mathrm{i}_{\mathrm{k}-1} \mathrm{D}^{-1}+\cdots+\mathrm{i}_{\mathrm{k}-\mathrm{L}} \mathrm{D}^{-\mathrm{L}}+\cdots+\mathrm{i}_{\mathrm{k}-\nu+1} \mathrm{D}^{-\nu+1} .
$$

Next, we compute the corresponding abstract state:

$$
\mathrm{v} \equiv\left(\mathrm{v}_{1}, \mathrm{v}_{2}\right)=(\psi \mathrm{G}) \mathrm{Q} \text {, }
$$

where "Q" is the projection operator that trancates sequences to start at time 1 :

$$
\begin{aligned}
\mathrm{v}_{1} & =\left(\psi \mathrm{g}^{(1)}(\mathrm{D})\right) \mathrm{Q}, \\
\mathrm{v}_{2} & =\left(\psi \mathrm{g}^{(2)}(\mathrm{D})\right) \mathrm{Q}=\left(\psi\left(\mathrm{g}^{(1)}(\mathrm{D})+\mathrm{D}^{\mathrm{L}}\right)\right) \mathrm{Q} \\
& =\mathrm{v}_{1}+\left(\psi \mathrm{D}^{\mathrm{L}}\right) \mathrm{Q} \\
& ={ }_{\mathrm{v}_{1}}+\mathrm{i}_{\mathrm{k}} \mathrm{D}^{\mathrm{L}}+\mathrm{i}_{\mathrm{k}-1} \mathrm{D}^{\mathrm{L}-1}+\cdots+\mathrm{i}_{\mathrm{k}-\mathrm{L}+1} \mathrm{D} .
\end{aligned}
$$

Finally, in order to transform the resulting abstract state to the syndrome former state (i.e., dual state), we compute $\mathrm{vH}^{\mathrm{T}}$. After some calculations we have

$$
\begin{aligned}
v^{\mathrm{T}}= & \left(v_{1}, v_{2}\right)\left(g^{(2)}(D), g^{(1)}(D)\right)^{\mathrm{T}} \\
= & v_{1}\left(g^{(1)}(D)+D^{\mathrm{L}}\right) \\
& +\left(v_{1}+i_{k} D^{\mathrm{L}}+i_{k-1} D^{L-1}+\cdots+i_{k-L+1} D\right) g^{(1)}(D) \\
= & v_{1} D^{L}+\left(i_{k} D^{L}+i_{k-1} D^{L-1}+\cdots+i_{k-L+1} D\right) g^{(1)}(D) \\
= & \cdots+i_{k-L} D^{\nu} .
\end{aligned}
$$

That is, the coefficient of $D^{\nu}$ is equal to $i_{k-L}$. Hence, the physical state of the syndrome former is expressed as $\sigma$ $(\mathrm{k})=\left(\sigma_{1}(\mathrm{k}), \sigma_{2}(\mathrm{k}), \cdots, \mathrm{i}_{\mathrm{k}-\mathrm{\nu}}\right)$.

It has been shown that the state $\left(\mathrm{i}_{\mathrm{k}-{ }_{\nu}+1}, \cdots, \mathrm{i}_{\mathrm{k}-\mathrm{L}}, \cdots, \mathrm{i}_{\mathrm{k}}\right)$ of the encoder corresponds to the state $\left(\sigma_{1}(\mathrm{k}), \sigma_{2}(\mathrm{k}), \cdots\right.$, $\mathrm{i}_{\mathrm{k}-\mathrm{L}}$ ) of the syndrome former. This fact means that the reliability of $\sigma_{\nu}(\mathrm{k})=\mathrm{e}_{\mathrm{k}}{ }^{(1)}+\mathrm{e}_{\mathrm{k}}{ }^{(2)}$, which can be obtained using bidirectional syndrome decoding, is identical to that of $i_{k-L}$. This is consistent with the result of Section 3 .

\section{Structure of Bidirectional Syndrome Decoding}

In this section, we examine the structure of bidirectional syndrome decoding introduced in the preceding section. In the following, we deal with a binary rate $(n-1) / n$, memory length $\nu$, convolutional code specified by a polynomial generator matrix $G(D)$. By calculating an invariant-factor decomposition [16], we get the corresponding syndrome former $\mathrm{H}^{\mathrm{T}}$. From the assumption of the code rate, $H^{T}$ has the form $H=\left(h_{1}(D), h_{2}(D), \cdots\right.$, $h_{n}(D)$ ). If both $G$ and $H$ are "minimal" , their overall constraint lengths are equal (i.e., $\nu$ ) [15], [16]. Then the maximum degree of $h_{j}(D)$ is $\nu$. Hence, an observer canonical form of the syndrome former $\mathrm{H}^{\mathrm{T}}$ contains $\nu$ memory elements.

Now, consider bidirectional syndrome decoding based on the syndrome trellis of $\mathrm{H}^{\mathrm{T}}[17]$. It is natural that backward decoding is also performed based on the syndrome trellis of $\mathrm{H}^{\mathrm{T}}$. In this case, however, it becomes necessary to generate the syndrome sequence in timereversed order for backward decoding. Because in order for the backward decoder to go backward, the corresponding syndrome bits must be delivered to it in advance.

Now, with each convolutional code $\mathrm{C}$ a reciprocal dual code $\mathrm{C}^{* \perp}$ can be associated. A generator matrix $\mathrm{H}^{*}$ of $\mathrm{C}^{* \perp}$ is obtained in the following way [12]:

(1) Substitute $D^{-1}$ for $D$ in the generator matrix $H(D)$ of the dual code $\mathrm{C}^{\perp}$.

(2) Then multiply the $\mathrm{j}$-th row of the resulting matrix with $D^{\gamma(0)}$, where $\gamma(j)$ is the degree of the $j$-th row of $\mathrm{H}(\mathrm{D})$.

Then it is shown that the time-reversed syndrome sequence is generated using $\mathrm{H}^{*}$.

Let $\left(e_{1}, \cdots, e_{k}, \cdots, e_{N}, e_{N+1}, \cdots, e_{N+}\right)$ be an error sequence, where $e_{k}=\left(e_{k}^{(1)}, \cdots, e_{k}^{(n)}\right)$ and $\left(e_{N^{+}}, \cdots, e_{N^{+}}\right)$are $\nu X_{n}$ " 0 " error symbols which force the syndrome former $\mathrm{H}^{\mathrm{T}}$ into the all-zero state. Then, the syndrome sequence $\zeta^{+} \equiv\left(\zeta_{1}, \cdots, \zeta_{k-1}, \zeta_{k}\right)$ is generated when the error symbols $\mathrm{e}^{+} \equiv\left(\mathrm{e}_{1}, \cdots, \mathrm{e}_{\mathrm{k}-{ }_{\nu}+1}, \cdots, \mathrm{e}_{\mathrm{k}-1}, \mathrm{e}_{\mathrm{k}}\right)$ are inputted to the syndrome former $\mathrm{H}^{\mathrm{T}}$. Similarly, the syndrome sequence $\zeta^{-} \equiv\left(\zeta_{\mathrm{N}^{+} \nu}, \cdots, \zeta_{\mathrm{k}+2}, \zeta_{\mathrm{k}+1}\right)$ is generated when the error symbols $\mathrm{e}^{-} \equiv\left(\mathrm{e}_{\mathrm{N}}, \cdots, \mathrm{e}_{\mathrm{k}}, \mathrm{e}_{\mathrm{k}-1}, \cdots, \mathrm{e}_{\mathrm{k}-{ }_{\nu}+1}\right)$ are inputted to the syndrome former $\mathrm{H}^{\mathrm{*} T}$.

Note that the two syndrome sequences $\zeta^{+}$and $\zeta^{-}$are disjoint and the corresponding two error sequences $e^{+}$and $e^{-}$have the intersection $\left(e_{k-{ }_{\nu}+1}, \cdots, e_{k-1}, e_{k}\right)$. Therefore, it has been shown that if the syndrome bits are generated once and only once using the forward syndrome former $\mathrm{H}^{\mathrm{T}}$ and the backward syndrome former $\mathrm{H}^{* \mathrm{~T}}$, then the corresponding two error sequences have the intersection 
of $\nu X_{n}$ error symbols. This property is considered to be dual to that for bidirectional (Viterbi) decoding based on a code trellis [6].

\section{Conclusions}

In this paper, we reviewed the Soft-BIVA based on the bidirectional Viterbi algorithm and stated its basic properties. Next, we applied the Soft-BIVA to general QLI codes. For QLI codes, in addition to conventional Viterbi decoding, SST Viterbi decoding can be applied. It has been shown that for SST Viterbi decoding of QLI codes, the reliability of the original information symbol $i_{k-L}$ can be obtained from that of the apparent information symbol $u_{k}$ for the main decoder. Furthermore, noting the close relation between SST Viterbi decoding and syndrome decoding for QLI codes, it has been shown that the reliability of $u_{k}$ can be obtained using bidirectional syndrome decoding. It has also been found that the syndrome sequence must be generated in time-reversed order for backward syndrome decoding and for this purpose a generator matrix of the reciprocal dual code of the original code is required.

\section{Acknowledgment}

The authors wish to thank Prof. Shigeich Hirasawa of Waseda University for his support during the course of this work. This work was supported in part by the Grant-in-Aid for Scientific Research of the Ministry of Education, Science and Culture of Japan under Grant A1-07558168.

\section{References}

[1] L. R. Bahl, J. Cocke, F. Jelinek, and J. Raviv, “Optimal decoding of linear codes for minimizing symbol error rate," IEEE Trans. Inform.Theory, vol.IT-20, no.2, pp.284-287, March 1974.

[2] G. D. Forney, Jr., "The Viterbi algorithm," Proc. IEEE, vol.61, no.3, pp.268-278, March 1973.

[3] C. Berrou, A. Glavieux and P. Thitimajshima, "Near Shannon limit error-correcting coding and decoding : Turbo-codes (1)," Proc. ICC’93, pp.1064-1070, May 1993.

[4] J. Hagenauer, E. Offer and L. Papke, "Iterative decoding of binary block and convolutional codes," IEEE Trans. Inform.Theory, vol.42, no.2, pp.429-445, March 1996.
[5] X. Wang and S. B. Wicker, "A soft-output decoding algorithm for concatenated systems," IEEE Trans. Inform.Theory, vol.42, no.2, pp.543-553, March 1996. [6] J. Belzile and D. Haccoun, "Bidirectional breadth-first algorithms for the decoding of convolutional codes," IEEE Trans. Commun., vol.41, no.2, pp.370-380, Feb. 1993.

[7] M. Tajima, "Bidirectional Viterbi decoding algorithm and its applications," (in Japanese), Technical Report of IEICE, IT93-110, pp.25-30, March 1994.

[8] M. Tajima, S. Suzuki and Z. Kawasaki, "Bidirectional Viterbi decoding algorithm with soft-outputs," (in Japanese), Technical Report of IEICE, IT96-80, pp.115-120, March 1997.

[9] M. B. Shoemake and C. Heegard, "Computationally efficient turbo decoding with the bi-directional Viterbi algorithm (BIVA)," Proc. ISIT'97, p.228, June/July 1997.

[10] J. Hagenauer and P. Hoeher, "A Viterbi algorithm with soft-decision outputs and its applications," Proc. GLOBECOM'89, pp.47.1.1.47.1.7.,Nov. 1989.

[11] S. Kubota, T. Kohri and S. Kato, "A SST (Scarce State Transition) type Viterbi decoder," (in Japanese), Trans. IECE, vol.J68-B, no.1, pp.38-45, Jan. 1985.

[12] S. Riedel, "Symbol-by-symbol MAP decoding of convolutional codes using reciprocal dual convolutional codes," Proc. ISIT'97,p.226, June/July 1997.

[13] M. Tajima, H. Suzuki and K. Kobayashi, "Saving Viterbi decoder states for general rate $1 / 2$, constraint length K, Quick Look-In codes," Electronics and Communications in Japan, Part 3, vol.73, no.11, pp.69-77, Nov. 1990.

[14] M. Tajima, "On the soft-decision metrics for SST Viterbi decoding of QLI codes," (in Japanese), Trans. IEICE, vol.J80-A, no.4, pp.708-711, April 1997.

[15] G. D. Forney, Jr., "Structural analysis of convolutional codes via dual codes," IEEE Trans. Inform.Theory,vol.IT-19, no.4, pp.512-518, July 1973.

[16] G. D. Forney, Jr., “Convolutional codes I : Algebraic structure," IEEE Trans. Inform.Theory, vol.IT-16, no.6, pp.720-738, Nov. 1970.

[17] J. P. M. Schalkwijk, A. J. Vinck and K. A. Post, "Syndrome decoding of binary rate $\mathrm{k} / \mathrm{n}$ convolutional codes," IEEE Trans. Inform.Theory, vol.IT-24, no.5, pp.553-562, Sept. 1978. 